\title{
Positive solutions for semilinear elliptic systems with sign-changing potentials
}

\author{
Noureddine Zeddini and Adel Ben Dkhil
}

\begin{abstract}
In this paper, we study the existence of positive solutions of the Dirichlet problem $-\Delta u=\lambda p(x) f(u, v) ;-\Delta v=\lambda q(x) g(u, v), \quad$ in $D$, and $u=v=0 \quad$ on $\partial^{\infty} D$, where $D \subset \mathbb{R}^{n}(n \geq 3)$ is an $C^{1,1}$-domain with compact boundary and $\lambda>0$. The potential functions $p, q$ are not necessarily bounded, may change sign and the functions $f, g: \mathbb{R}^{2} \rightarrow \mathbb{R}$ are continuous with $f(0,0)>0, g(0,0)>0$. By applying the LeraySchauder fixed point theorem, we establish the existence of positive solutions for $\lambda$ sufficiently small.
\end{abstract}

\section{Introduction}

Let $D$ be a $C^{1,1}$ domain of $\mathbb{R}^{n}(n \geq 3)$ with compact boundary and let $\partial^{\infty} D=$ $\partial D$ if $D$ is bounded and $\partial^{\infty} D=\partial D \cup\{\infty\}$ whenever $D$ is unbounded. This paper deals with the existence of positive continuous solutions (in the sense of distributions) for the following semilinear elliptic system

$$
\left\{\begin{array}{l}
-\Delta u=\lambda p(x) f(u, v), \quad \text { in } D \\
-\Delta v=\lambda q(x) g(u, v), \quad \text { in } D \\
u=v=0 \quad \text { on } \partial^{\infty} D,
\end{array}\right.
$$

where the potential functions $p, q$ are sign changing functions belonging to the Kato class $K(D)$ introduced and studied in [1] and [9] and $f, g$ satisfy the following hypothesis.

Key Words: Elliptic Systems, Positive Solution, Green potential, Leray-Shauder fixed point theorem

2010 Mathematics Subject Classification: Primary 35J47,35J57; Secondary 35J08,35P30. Received: 24.01.2014

Accepted: 3.09.2014. 
$\left(\mathbf{H}_{\mathbf{1}}\right)$ The functions $f, g: \mathbb{R}^{2} \rightarrow \mathbb{R}$ are continuous with $f(0,0)>0$ and $g(0,0)>0$.

In recent years, a good amount of research is established for reaction-diffusion systems. reaction-diffusions systems model many phenomena in Biology, Ecology, combustion theory, chemical reactors, population dynamics etc. And the case $p(x)=q(x)=1$ has been considered as a typical example when $D$ is a bounded regular domain in $\mathbb{R}^{n}$ and many existence results where established by variational methods, topological methods and the method of sub and supersolution (see [5], [7], [4]).

Recently, Chen [2] studied the existence of positive solutions for the following system

$$
\left\{\begin{array}{l}
-\Delta u=\lambda p(x) f_{1}(v), \quad \text { in } D \\
-\Delta v=\lambda q(x) g_{1}(v), \quad \text { in } D, \\
u=v=0 \quad \text { on } \partial^{\infty} D,
\end{array}\right.
$$

where $D$ is a bounded domain. He assumed that if $p, q$ are continuous in $\bar{D}$ and

$\left(\mathbf{H}_{2}\right)$ There exists $\mu_{1}, \mu_{2}>0$ such that

$$
\begin{aligned}
& \int_{D} G(x, y) p_{+}(y) d y>\left(1+\mu_{1}\right) \int_{D} G(x, y) p_{-}(y) d y \quad \forall x \in D \\
& \int_{D} G(x, y) q_{+}(y) d y>\left(1+\mu_{2}\right) \int_{D} G(x, y) q_{-}(y) d y \quad \forall x \in D
\end{aligned}
$$

where $G(x, y)$ is the Green's function of the Dirichlet Laplacian in $D$. Here $p^{+}, q^{+}$are the positive parts of $p$ and $q$, while $p_{-}, q_{-}$are the negative ones.

The main result of Chen [2] reads as follows.

Theorem A. Let $p, q$ be nonzero continuous functions on $\bar{D}$ satisfying $\mathbf{H}_{\mathbf{2}}$ ) and let $f_{1}, g_{1}:[0, \infty) \rightarrow \mathbb{R}$ be continuous with $f_{1}(0)>0, g_{1}(0)>0$. Then there exists a positive number $\lambda^{\star}>0$ such that (1.2) has a positive solution for $0<\lambda<\lambda^{\star}$.

We note that in the case where $f_{1}, g_{1}$ are nonnegative nondecreasing continuous functions, $p(x) \leq 0$ in $D$ and $q(x) \leq 0$ in $D$, system (1.2) was studied in $[6]$ with nontrivial nonnegative boundary data and the existence of positive bounded solutions for (1.2) was established whenever $\lambda$ is a small positive real number.

Our aim in this paper is to extend and improve, by a modified proof, the result of Chen [2] in a number of ways. First, the domain $D$ will be bounded or an exterior domain. Second, the functions $p, q$ are not necessarily continuous in $\bar{D}$. 
Indeed $p, q$ may be singular on the boundary of $D$. Third, the nonlinear terms $f_{1}(v)$ and $g_{1}(u)$ considered in [2] are more restrictive than the class $f(u, v)$ and $g(u, v)$ considered in our case. More precisely, we will establish the existence of a positive solution for (1.1) in the case where $f(0,0)>0, g(0,0)>0$ and the potentials of $p, q$ satisfy hypothesis $\left(\mathbf{H}_{\mathbf{2}}\right)$ and belong to the Kato class introduced and studied in [1] and [9]. A nonexistence of positive bounded solution will be also given in the case where $f$ and $g$ are sublinear functions with $f(0,0)=0$ and $g(0,0)=0$. To this aim, we give in the sequel some notations and we recall some properties of the Kato class.

Definition 1.1. (See [1] and [9].) A Borel measurable function $k$ in $D$ belongs to the Kato class $K(D)$ if

$$
\lim _{\alpha \rightarrow 0} \sup _{x \in D} \int_{D \cap B(x, \alpha)} \frac{\rho(y)}{\rho(x)} G(x, y)|k(y)| d y=0
$$

and satisfies further

$\lim _{M \rightarrow \infty} \sup _{x \in D} \int_{D \cap\{|y| \geq M\}} \frac{\rho(y)}{\rho(x)} G(x, y)|k(y)| d y=0 \quad$ ( whenever $D$ is unbounded),

where $\rho(x)=\min (1, \delta(x))$ and $\delta(x)$ denotes the euclidian distance from $x$ to the boundary of $D$.

We remark that in the case where $D$ is bounded an if $d$ denotes its diameter, then

$$
\frac{1}{1+d} \delta(x) \leq \rho(x) \leq \delta(x)
$$

So in this case, we can replace $\rho(x)$ by $\delta(x)$ in the Definition 1.1 .

Next, we give some examples of functions belonging to $K(D)$.

Example 1.1. (see [1] and [9])

(1) Let $D$ be a bounded domain of $\mathbb{R}^{n}$.

(a) Let $q(y)=\frac{1}{(\delta(x))^{\lambda}}$. Then $q \in K(D)$ if and only if $\lambda<2$.

(b) Let $p>\frac{n}{2}$, then for $\lambda<2-\frac{n}{p}$, we have $\frac{1}{\delta(.)^{\lambda}} L^{p}(D) \subset K(D)$. In particular $\quad L^{p}(D) \subset K(D)$.

(c) Let $D=B(0,1)$ and let $q$ be a Borel radial function in $D$, then $q \in K(D) \quad$ if and only if $\int_{0}^{1} r(1-r)|q(r)| d r<\infty$.

(2) Let $D$ be a $C^{1,1}$-exterior domain in $\mathbb{R}^{n}(n \geq 3)$. The function $x \rightarrow$ $\frac{1}{(|x|+1)^{\mu-\lambda} \delta(x)^{\lambda}} \in K(D)$ if and only if $\lambda<2<\mu$. 
(3) Let $D=\overline{B(0,1)^{c}}$ be the exterior of the unit closed ball in $\mathbb{R}^{n}(n \geq 3)$ and let $q$ be a Borel radial function in $D$, then $q \in K(D)$ if and only if $\int_{1}^{\infty}(r-1)|q(r)| d r<\infty$.

For any nonnegative Borel measurable function $\varphi$ in $D$, we denote by $V \varphi$ the Green potential of $\varphi$ defined on $D$ by

$$
V \varphi(x)=\int_{D} G(x, y) \varphi(y) d y
$$

Recall that if $\varphi \in L_{l o c}^{1}(D)$ and $V \varphi \in L_{l o c}^{1}(D)$, then we have in the distributional sense (see [3] p. 52)

$$
\Delta(V \varphi)=-\varphi \text { in } D
$$

Our main results are as follows.

Theorem 1.2. Let $p, q$ be in the Kato class $K(D)$ and assume that hypotheses $\left(\mathbf{H}_{1}\right)-\left(\mathbf{H}_{2}\right)$ are satisfied. Then there exists $\lambda_{0}>0$ such that for each $\lambda \in$ $\left(0, \lambda_{0}\right)$, problem (1.1) has a positive continuous solution in $D$.

For the nonexistence of positive bounded solutions, we establish

Theorem 1.3. Let $p, q$ be two nontrivial functions in the Kato class $K(D)$. Assume that the functions $f, g: \mathbb{R}^{2} \rightarrow \mathbb{R}$ are measurable and there exists a positive constant $M$ such that for all $u, v$ we have,

$$
\begin{aligned}
& |f(u, v)| \leq M(|u|+|v|) \\
& |g(u, v)| \leq M(|u|+|v|) .
\end{aligned}
$$

Then there exists $\lambda_{0}>0$ such that the problem (1.1) has no bounded positive continuous solution in $D$ for each $\lambda \in\left(0, \lambda_{0}\right)$.

Throughout this paper, we denote by $B(D)$ the set of Borel measurable functions in $D$ and by $C_{0}(D)$ the set of continuous ones satisfying $\lim _{x \rightarrow \xi \in \partial^{\infty} D} u(x)=0$.

Finally, for a bounded real function $\omega$ defined on a set $S$ we denote by $\|\omega\|_{\infty}=$ $\sup _{x \in S}|\omega(x)|$.

\section{Proof of Theorems 1.2 and 1.3}

We begin this section by giving a continuity result.

Proposition 2.1. (see [1] and [9]) Let $\varphi$ be a nonnegative function in $K(D)$. Then we have 
i) The function $y \rightarrow \frac{\delta(y)}{(1+|y|)^{n-1}} \varphi(y)$ is in $L^{1}(D)$. In particular $\varphi \in L_{l o c}^{1}(D)$.

ii) $V \varphi \in C_{0}(D)$.

iii) Let $h_{0}$ be a positive harmonic function in $D$ which is continuous and bounded in $\bar{D}$. Then the family of functions

$$
\left.\left\{\int_{D} G ., y\right) h_{0}(y) p(y) d y:|p| \leq \varphi\right\}
$$

is relatively compact in $C_{0}(D)$.

Next, we recall first the Leray-Schauder fixed point theorem.

Lemma 2.2. (Leray-Schauder fixed point theorem) Let $X$ be a Banach space with norm $\|$.$\| and x_{0}$ be a point of $X$. Suppose that $T: X \times[0,1] \rightarrow X$ is continuous and compact with $T(x, 0)=x_{0}$, for each $x \in X$, and there exists a fixed constant $M>0$ such that each solution $(x, \sigma) \in X \times[0,1]$ of the $T(x, \sigma)=x$ satisfies $\|x\| \leq M$. Then $T(., 1)$ has a fixed point.

Using this Lemma, we obtain the following general existence result.

Lemma 2.3. Suppose that $p$ and $q$ are in the Kato class $K(D)$ and $f, g$ are continuous and bounded from $\mathbb{R}^{2}$ to $\mathbb{R}$. Then for every $\lambda \in(0, \infty)$, problem (1.1) has a solution $\left(u_{\lambda}, v_{\lambda}\right) \in C_{0}(D) \times C_{0}(D)$.

Proof. For $\lambda \in \mathbb{R}$, we consider the operator $T_{\lambda}: C_{0}(D) \times C_{0}(D) \times[0,1] \rightarrow$ $C_{0}(D) \times C_{0}(D)$ defined by

$$
T_{\lambda}((u, v), \sigma)=(\sigma \lambda V(p f(u, v)), \sigma \lambda V(q g(u, v))) .
$$

By Proposition 2.1, the operator $T_{\lambda}$ is well defined, continuous, compact and $T_{\lambda}((u, v), 0)=(0,0):=x_{0} \in C_{0}(D) \times C_{0}(D)$. Let $(u, v) \in C_{0}(D) \times C_{0}(D)$ and $\sigma \in[0,1]$ such that $T_{\lambda}((u, v), \sigma)=(u, v)$. Then, since $f, g$ are bounded and $p$, $q$ are in $K(D)$ we deduce by using Proposition 2.1 that

$$
\begin{aligned}
\max \left(\|u\|_{\infty},\|v\|_{\infty}\right) & =\sigma \lambda \max \left(\|V(p f(u, v))\|_{\infty},\|V(q g(u, v))\|_{\infty}\right) \\
& \leq \lambda \max \left(\|V p\|_{\infty}\|f\|_{\infty},\|V q\|_{\infty}\|g\|_{\infty}\right) \\
& =M
\end{aligned}
$$

Hence by Leray-Schauder fixed point theorem, the operator $T_{\lambda}(., 1)$ has a fixed point. Namely, there exists $(u, v) \in C_{0}(D) \times C_{0}(D)$ such that $(u, v)=$ $(\lambda V(p f(u, v)), \lambda V(q g(u, v)))$. So, using (1.3) and Proposition 2.1, we deduce that $(u, v)$ is a solution of system (1.1). 
Proof of Theorem 1.2. Fix a large number $M>0$ and an infinitely continuously differentiable function $\psi$ with compact support on $\mathbb{R}^{2}$ such that $\psi=1$ in the open ball with center 0 and radius $M$ and $\psi=0$ on the exterior of the ball with center 0 and radius $2 M$. Define the bounded functions $\widetilde{f}, \widetilde{g}$ on $\mathbb{R}^{2}$ by $\widetilde{f}(u, v)=\psi(u, v) f(u, v)$ and $\widetilde{g}(u, v)=\psi(u, v) g(u, v)$. By Lemma 2.3, the Dirichlet problem:

$$
\left\{\begin{array}{l}
-\Delta u=\lambda p(x) \tilde{f}(u, v), \quad \text { in } D, \\
-\Delta v=\lambda q(x) \widetilde{g}(u, v), \quad \text { in } D, \\
u=v=0 \quad \text { on } \partial^{\infty} D,
\end{array}\right.
$$

has a solution $\left(u_{\lambda}, v_{\lambda}\right) \in C_{0}(D) \times C_{0}(D)$ satisfying

$$
\left(u_{\lambda}, v_{\lambda}\right)=\left(\lambda V\left(p \widetilde{f}\left(u_{\lambda}, v_{\lambda}\right)\right) \lambda V\left(q \widetilde{g}\left(u_{\lambda}, v_{\lambda}\right)\right)\right) .
$$

Moreover

$$
\max \left(\left\|u_{\lambda}\right\|_{\infty},\left\|v_{\lambda}\right\|_{\infty}\right) \leq \lambda \max \left(\|V p\|_{\infty}\|\widetilde{f}\|_{\infty},\|V q\|_{\infty}\|\widetilde{g}\|_{\infty}\right)
$$

Put $\mu=\min \left(\mu_{1}, \mu_{2}\right)$ and consider $\gamma \in\left(0, \frac{\mu}{2+\mu}\right)$. Since $\tilde{f}$ and $\widetilde{g}$ are continuous, then there exists $\delta \in(0, M)$ such that if $\max (|\zeta|,|\xi|)<\delta$, we have $\tilde{f}(0,0)(1-$ $\gamma)<\widetilde{f}(\zeta, \xi)<\widetilde{f}(0,0)(1+\gamma)$ and $\widetilde{g}(0,0)(1-\gamma)<\widetilde{g}(\zeta, \xi)<\widetilde{g}(0,0)(1+\gamma)$.

Using (2.2), we deduce that there exists $\lambda_{0}>0$ such that $\left\|u_{\lambda}\right\|_{\infty}<\delta$ and $\left\|v_{\lambda}\right\|_{\infty}<\delta$ for any $\lambda \in\left(0, \lambda_{0}\right)$. This together with the fact that $0<\delta<M$ implies that for $\lambda \in\left(0, \lambda_{0}\right)$, we have $\widetilde{f}\left(u_{\lambda}, v_{\lambda}\right)=f\left(u_{\lambda}, v_{\lambda}\right)$ and $\widetilde{g}\left(u_{\lambda}, v_{\lambda}\right)=$ $g\left(u_{\lambda}, v_{\lambda}\right)$.

Now, for each $x \in D$ we have

$$
\begin{aligned}
u_{\lambda} & =\lambda V\left(p_{+} \tilde{f}\left(u_{\lambda}, v_{\lambda}\right)\right)-\lambda V\left(p_{-} \tilde{f}\left(u_{\lambda}, v_{\lambda}\right)\right) \\
& >\lambda f(0,0)(1-\gamma) V\left(p_{+}\right)-\lambda f(0,0)(1+\gamma) V\left(p_{-}\right) \\
& >\lambda f(0,0)\left[(1-\gamma)\left(1+\mu_{1}\right)-(1+\gamma)\right] V\left(p_{-}\right) \\
& >\lambda f(0,0)(1-\gamma)\left[1+\mu_{1}-\frac{1+\gamma}{1-\gamma}\right] V\left(p_{-}\right) \\
& >\lambda f(0,0)(1-\gamma)\left[1+\mu-\frac{1+\gamma}{1-\gamma}\right] V\left(p_{-}\right) .
\end{aligned}
$$

Now, since $\gamma \in\left(0, \frac{\mu}{2+\mu}\right)$, then $1+\mu-\frac{1+\gamma}{1-\gamma}>0$ and it follows that $\lambda f(0,0)(1-\gamma)\left[1+\mu-\frac{1+\gamma}{1-\gamma}\right] V\left(p_{-}\right) \geq 0$. Consequently, for each $\lambda \in\left(0, \lambda_{0}\right)$ and for each $x \in D$ we have $u_{\lambda}(x)>0$. Similarly, we obtain $v_{\lambda}(x)>0$ for each $x \in D$. 
Proof of Theorem 1.3 Suppose that (1.1) has a bounded positive solution $(u, v)$ for $\lambda>0$. Then $f(u, v)$ and $g(u, v)$ are bounded. Put $\widetilde{u}=\lambda V(p f(u, v))$ and $\widetilde{v}=\lambda V(q g(u, v))$. Since $f(u, v)$ and $g(u, v)$ are bounded, then the functions $\widetilde{u}, \widetilde{v} \in C_{0}(D)$. The functions $z=u-\widetilde{u}$ and $\omega=v-\widetilde{v}$ are harmonic in the distributional sense and continuous in $D$, so they are harmonic in the classical sense. Moreover, since $u=\widetilde{u}=v=\widetilde{v}=0$ on $\partial^{\infty} D$ then $u=\widetilde{u}$ and $v=\widetilde{v}$ in $D$. Which implies

$$
\begin{gathered}
\|u\|_{\infty} \leq \lambda V(|p| f(u, v)) \leq \lambda M\|V(|p|)\|_{\infty}\left(\|u\|_{\infty}+\|v\|_{\infty}\right), \\
\|v\|_{\infty} \leq \lambda V(|q| g(u, v)) \leq \lambda M\|V(|q|)\|_{\infty}\left(\|u\|_{\infty}+\|v\|_{\infty}\right) .
\end{gathered}
$$

By adding these inequalities, we obtain

$$
\left(\|u\|_{\infty}+\|v\|_{\infty}\right) \leq \lambda M\left[\|V(|p|)\|_{\infty}+\|V(|q|)\|_{\infty}\right]\left(\|u\|_{\infty}+\|v\|_{\infty}\right) .
$$

This gives a contradiction if $\lambda M\left[\|V(|p|)\|_{\infty}+\|V(|q|)\|_{\infty}\right]<1$.

Example 2.1. Let $p, q$ be two measurable radial functions on the exterior of the unit ball $D=\overline{B(0,1)}^{c}, n \geq 3$. Assume that there exists $\varepsilon>0$ such that each $t>1$ and $x \in D$, we have

$$
\begin{aligned}
& \int_{1}^{t} \frac{r^{n-1}}{(|x| \vee r)^{n-2}}\left(1-(|x| \wedge r)^{2-n}\right) p^{+}(r) d r \\
& \quad \geq(1+\varepsilon) \int_{1}^{t} \frac{r^{n-1}}{(|x| \vee r)^{n-2}}\left(1-(|x| \wedge r)^{2-n}\right) p^{-}(r) d r, \quad \text { and } \\
& \int_{1}^{t} \frac{r^{n-1}}{(|x| \vee r)^{n-2}}\left(1-(|x| \wedge r)^{2-n}\right) q^{+}(r) d r \\
& \geq(1+\varepsilon) \int_{1}^{t} \frac{r^{n-1}}{(|x| \vee r)^{n-2}}\left(1-(|x| \wedge r)^{2-n}\right) q^{-}(r) d r
\end{aligned}
$$

then hypothesis $\left(\mathbf{H}_{\mathbf{2}}\right)$ is satisfied. Indeed (see [1]), for a nonnegative radial function $k$, the function $x \rightarrow \int_{D} G_{D}(x, y) k(|y|) d y$ is radial and

$$
\int_{D} G_{D}(x, y) k(|y|) d y=a_{n} \int_{1}^{\infty} \frac{r^{n-1}}{(|x| \vee r)^{n-2}}\left(1-(|x| \wedge r)^{2-n}\right) k(r) d r,
$$

where $|x| \wedge t=\min (|x|, t),|x| \vee t=\max (|x|, t)$ and $a_{n}>0$.

\section{References}

[1] I. Bachar, H. Mâagli, N. Zeddini, Estimates on the Green function and existence of positive solutions of nonlinear singular elliptic equations, Commun. Contemp. Math. 5(3) (2003) 401-434. 
[2] Ruipeng Chen, Existence of positive solutions for semilinear elliptic systems with indefinite weight, Electronic Journal of Differential Equations. 164 (2011) 1-8.

[3] K.L. Chung, Z. Zhao, From Brownian motion to Schrodinger's equation, Springer Verlag 1995.

[4] R. Dalmasso, Existence and uniqueness of positive solutions of semilinear elliptic systems, Nonlinear Anal. 39 (2000), 559-568.

[5] D. G. de Figueiredo, J. Marcos do O, B. Ruf, An Orlicz space approach to superlinear elliptic systems, J. Func. Anal. 224 (2005), pp. 471-496.

[6] A. Ghanmi, H. Mâagli, V. S. Turki, N. Zeddini, Existence of positive bounded solutions for some nonlinear elliptic systems, J. Math. Anal. Appl. 352(2009) 440-448.

[7] D. Hulshof, R. Van der Vorst, Differential systems with strongly indefinite variational structure, J. Func. Anal. 114 (1993), pp. 32-58.

[8] J. Tyagi, Existence of non-negative solutions for predator-prey elliptic systems with a sign-changing nonlinearity, Electronic Journal of Differential Equations. 153 (2011) 1-9.

[9] H. Mâagli, M. Zribi, On a new Kato class and singular solutions of a nonlinear elliptic equation in bounded domains, Positivity, 9 (2005) 667686.

[10] D. D. Hai, Positive solutions of elliptic boundary value problems, J. Math. Anal. Appl, 227 (1998), 195-199.

Noureddine Zeddini,

Department of Mathematics,

College of Sciences and Arts,

King Abdulaziz University, Rabigh Campus

Box 344, Rabigh 21911, Saudi Arabia.

Email: nalzeddini@kau.edu.sa noureddine.zeddini@ipein.rnu.tn

Adel Ben Dkhil,

Department of Mathematics,

Faculty of Sciences of Tunis,

University of Tunis El-Manar,

Campus Universitaire, 2092 Tunis, Tunisia.

Email:Adel.Bendekhil@ipein.rnu.tn 